\title{
Spiky Burst of High Frequency Heart Rate Variability: A Prodromal Sign of Syncope Accompanying Heatstroke
}

\author{
Emi Yuda, Rui Iino, Yutaka Yoshida, and Junichiro Hayano
}

\begin{abstract}
Heart rate variability in Holter electrocardiogram (ECG) that incidentally recorded the onset process of heatstroke during golf play was analyzed in comparison to control ECGs in the same subject and in a healthy control group. During playing golf normally, the amplitude of high-frequency (HF) component, known as an index of cardiac parasympathetic function, was suppressed $(4.3 \pm 2.2 \mathrm{~ms})$. Whereas, $135 \mathrm{~min}$ before the onset of syncope, spiky HF bursts (amplitude $\sim 10 \mathrm{~ms}$ and width $<60 \mathrm{~s}$ ) appeared repeatedly at an interval of 10-15 min and the burst amplitude increased to $20-30 \mathrm{~ms}$ from $51 \mathrm{~min}$ before to the onset of syncope. No such phenomenon was observed in control ECGs even during walking exercise. Although spiky HF burst was observed $30 \%$ of subjects in control group, none of them showed repetitive burst during short period $<15 \mathrm{~min}$. Repetitive spiky HF burst may be a prodromal sign for predicting heatstroke from heartbeat information.
\end{abstract}

Index Terms-Heatstroke, syncope, heart rate variability, autonomic nervous system, hot environment, golf, Holter electrocardiogram, complex demodulation.

\section{INTRODUCTION}

In recent years, as the temperature in summer rises with global warming, the risk of heatstroke during outdoor work and exercise is increasing [1], [2]. To prevent heatstroke and the accidents caused by it, it is important to develop technology to predict the onset of heatstroke for people who have to stay outside on hot days. Wearable sensors to monitor heart rate variability (HRV) and analysis of autonomic functions by it seem promising candidates because the autonomic controls of the cardiovascular system are critical mechanisms for heat adaptation [3], [4]. We have limited knowledge, however, on changes in HRV associated with heatstroke, particularly on changes preceding it.

Recently, we got an opportunity to analyze HRV in a case of accidental heatstroke occurring during golf playing under Holter electrocardiographic (ECG) monitoring. The data showed dynamic changes in HRV during the onset process of heatstroke leading syncope. We extracted features of HRV signs preceding the onset and investigated whether such signs

Manuscript received September 19, 2018; revised May 22, 2019. This work was conducted as a collaborative study between Belling Co., Ltd. and Nagoya City University Graduate School of Medical Sciences.

E. Yuda is with the Department of Electrical Engineering, Tohoku University Graduate School of Engineering, Sendai 980-8759, Japan (e-mail emi.yuda.a1@tohoku.ac.jp).

R. Iino is with Belling Co., Ltd. Kashiwa City 277-0822, Chiba, Japan

Y. Yoshida is with the Nagoya City University Graduate School of Design and Architecture, Nagoya 464-0083, Japan (e-mail: yoshida@sda.nagoya-cu.ac.jp).

J. Hayano is with the Department of Medical Education, Nagoya City University Graduate School of Medical Sciences, Nagoya 467-8602, Japan (e-mail: hayano@med.nagoya-cu.ac.jp). are specific to heatstroke by comparing the HRV data with those obtained on other days of exercise and golf playing without heatstroke symptom in the same subject and also those in a healthy control group.

To detect dynamic changes in HRV that cannot be obtained by power spectral analysis requiring a stationary state of data, we used complex demodulation (CDM) [5], [6] for HRV analysis. CDM is a time domain method that provides the time dependent changes in amplitude and frequency of specified fluctuation components as the functions of time. Fluctuation components of interest are specified as frequency bands, such as very-low frequency (VLF, $0.0033-0.4 \mathrm{~Hz}$ ), low frequency (LF, 0.04-0.15 Hz), and high frequency $(0.15-0.45 \mathrm{~Hz}$ ) bands. We observed repetitive "spiky bursts" of the amplitude of HF component during the period preceding syncope accompanying heatstroke.

\section{METHODS}

\section{A. Subject}

This is a case and control study. The case was a lady in her 30s who had been healthy and had no history of chronic disorders. She was explained the objectives, methods, and data collected for the study and she gave a written informed consent of participation. The control was a group of 70 healthy subjects consisting of 19 females and 51 males (age, $35 \pm 12 \mathrm{yr}$, range 21-60 year).

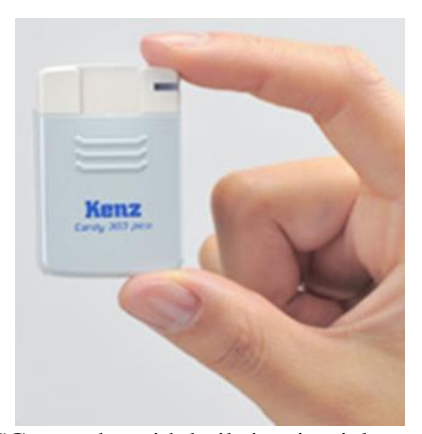

Fig. 1. Holter ECG recorder with built-in tri-axial acceleration sensors

This study was approved by the Ethics Review Committee of Nagoya City University Graduate School of Medicine and Nagoya City University Hospital (number 60160073).

\section{B. Measurements}

ECG and acceleration were measured with Holter ECG recorder with built-in tri-axial acceleration sensors (Cardy 303 pico+, Suzuken Co., Ltd., Nagoya, Japan). The size of the recorder was W28×D42×H9 mm and weight $13 \mathrm{~g}$ (Fig. 1). It recorded continuously 3-chanel ECG at $125 \mathrm{~Hz} /$ channel and tri-axial accelerations at $31.25 \mathrm{~Hz}$ for $24 \mathrm{~h}$ with a button electrode. 


\begin{tabular}{lccc}
\multicolumn{4}{c}{ TABLE I: WEATHER DATA OF THE DAYS OF GOLF PLAYING } \\
\hline & July & December & March \\
& 2017 & 2017 & 2018 \\
\hline Temperature & & & \\
Average, ${ }^{\circ} \mathrm{C}$ & 27.6 & 2.0 & 6.9 \\
Maximum, ${ }^{\circ} \mathrm{C}$ & 33.1 & 9.2 & 14.1 \\
Minimum, ${ }^{\circ} \mathrm{C}$ & 25.2 & -4.1 & 0.1 \\
Humidity, $\%$ & 45 & 62 & 48 \\
Atmospheric Pressure, hPa & 1006.0 & 1022.7 & 1020.5 \\
Sun shine, hr & 8.0 & 9.0 & 9.2 \\
\hline
\end{tabular}

\section{Protocol}

In the case subject, 24-h Holter ECG was recorded 6 times on three days of golf playing, two days of laboratory exercise test (walking and running), and a day of non-exercise control.

Golf playing: On a day out of three days of golf playing, she developed heatstroke accompanied by syncopal attack. The golf play was performed in July 2016 under Holter monitoring as a study aiming at investigating autonomic nervous function during golf play, during which heatstroke developed incidentally. The weather condition of the golf course was fine, the maximum temperature was $33.1^{\circ} \mathrm{C}$, and the humidity was $45 \%$ (Table I). The play started at 08:45 and had scheduled to finish at 15:00. She developed a heatstroke at $14: 40 \mathrm{pm}$ and interrupted play. On the other two days of golf playing performed on December 2017 and March 2018. In these days, she did not develop heatstroke or syncope.

Exercise tests: Treadmill exercise test were performed under Holter monitoring on two separate days in July 2017 in a laboratory for exercise test. For both days, the room temperature was set at $25{ }^{\circ} \mathrm{C}$. On one day, walking on a treadmill was started at 10:00 and carried out for $30 \mathrm{~min}$ at 4 $\mathrm{km} / \mathrm{h}$. On the other day, running on the treadmill was started at 10:00 and carried out for $30 \mathrm{~min}$ at $8 \mathrm{~km} / \mathrm{h}$.

Non-exercise control: Holter monitoring was performed on a day in July 2017 under usual daily activities without intended exercise.

Subjects in the control group underwent 24-h Holter ECG monitoring under their usual daily activities. During the monitoring, none of the subjects reported symptom or sign suggesting health problems including heatstroke or syncope.

\section{Analyses of ECG and Actigraphic Data}

The recorded ECG and actigraphic data were analyzed with Holter ECG scanner (Cardy Analyzer 05, Suzuken Co., Ltd., Nagoya, Japan), on which the temporal positions of all $\mathrm{R}$-waves were detected, the rhythm annotations were given to all QRS complexes, and all errors in automated analysis were edited manually. Actigraphic data were filed as acceleration to left-to-right, caudo-cranial, and postero-anterior axes as $x$, $y$, and $z$ values, respectively, at $31.25 \mathrm{~Hz}$.

The changes in R-R interval were analyzed by the method of complex demodulation (CDM) [5], [6]. For CDM analysis, $\mathrm{R}-\mathrm{R}$ interval time series were interpolated only using $\mathrm{R}-\mathrm{R}$ intervals consisting of consecutive sinus beats and resampled with equidistant time interval at $2 \mathrm{~Hz}$. Then, frequency bands of HRV including VLF, LF, and HF were demodulated by CDM one-by-one.

For actigraphic data, the changes in body posture were estimated from $\mathrm{x}, \mathrm{y}$, and $\mathrm{z}$ values. The subject was assumed to be in the lying position when the value of $y$ axis was below a threshold and to be in the upright position otherwise. Also, time series of $x(t), y(t)$, and $z(t)$ were high-pass filtered to remove direct current component caused by gravitational acceleration and combined into the vector magnitude (square root of the sum of $x(t), y(t)$, and $z(t)$ squares) as the estimate of physical activity, $A(t)$.

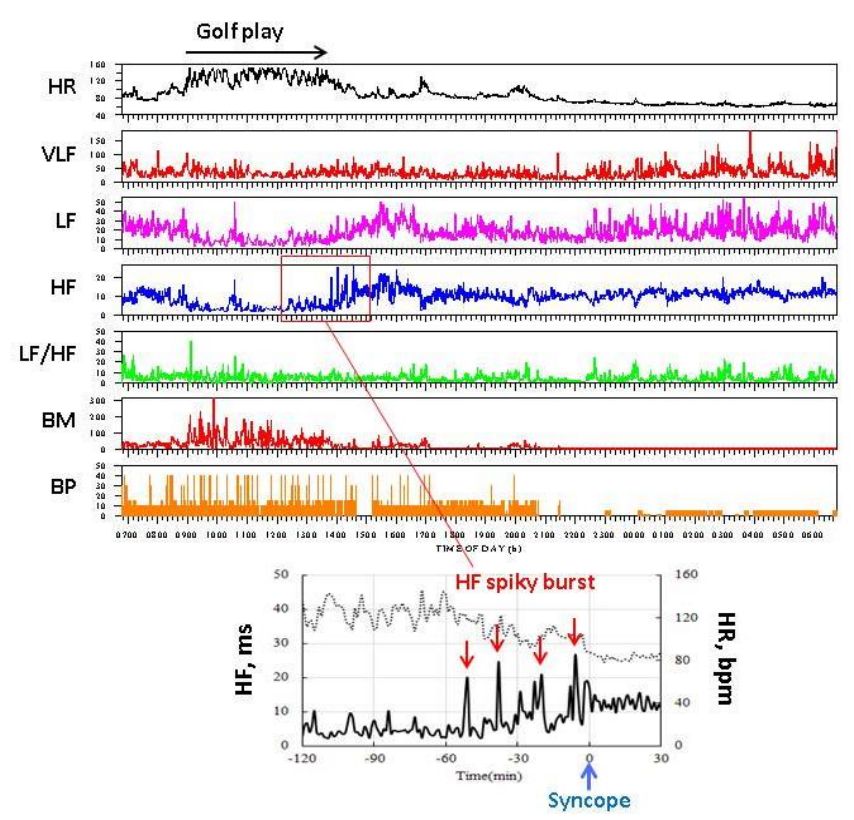

Fig. 2. Heart rate (HR), heart rate variability (HRV), body movement (BM), and body posture (BP) during $24 \mathrm{~h}$ in a subject who developed neurally mediated syncope (NMS) accompanying heatstroke during golf play (upper panels) and spiky burst of high frequency (HF) component of HRV observed before the episode of NMS (lower panel). HRV was analyzed by complex demodulation $(\mathrm{CDM}) . \mathrm{VLF}=$ very low frequency amplitude, $\mathrm{LF}=$ low frequency amplitude.

The VLF, LF, and HF amplitude of HRV and physical activity were averaged over every $1 \mathrm{~min}$. In the same 1 -min time frame, average heart rate and standard deviation of R-R intervals were calculated only using $\mathrm{R}-\mathrm{R}$ intervals consisting of consecutive sinus beats.

\section{E. Analyses of HRV Accompanying Heatstroke}

In a previous study of neurally mediated syncope (NMS) induced by head-up tilt test, we observed that HF amplitude of HRV increased about $90 \mathrm{sec}$ before the development of syncope [7]. Thus, we focused on the changes in HF amplitude before the development of the syncope accompanying heatstroke.

As shown in Fig. 2, HF amplitude showed "spiky bursts" (transient increase in HF amplitude for $\sim 1 \mathrm{~min}$ ) during $60 \mathrm{~min}$ before development of the syncope. Thus, we developed an algorithm to detect this phenomenon automatically (Fig. 3). We defined spiky burst ratio (SBR) as

$$
\mathrm{SBR}=((\mathrm{AV}(\mathrm{t}-\mathrm{w}, \mathrm{t}+\mathrm{w})-\mathrm{AVc}) / \mathrm{SDc},
$$

where

$$
\begin{aligned}
& A V c=\max (A V(t-2 w, t-w), A V(t+w, T+2 w)), \\
& S D c=\max (S D(t-2 w, t-w), S D(t+w, T+2 w)),
\end{aligned}
$$

and $A V(p, q)$ and $S D(p, q)$ are the average and standard 
deviation of HF amplitude from time point $p$ to time point $q$, respectively. We set $w$ at $60 \mathrm{sec}$ in this study and the occurrence of "spiky HF burst" was judged when SBR exceeded 4.0 .

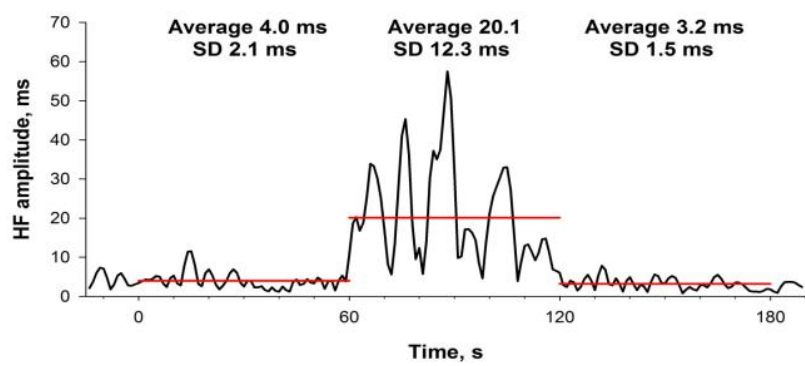

Fig. 3. Definition of spiky HF burst Spike HF burst is defined as transient increase in $\mathrm{HF}$ amplitude for $\sim 1$ min that is 4 standard deviations (SDs) greater than the HF amplitude during 1 min before and after the burst.

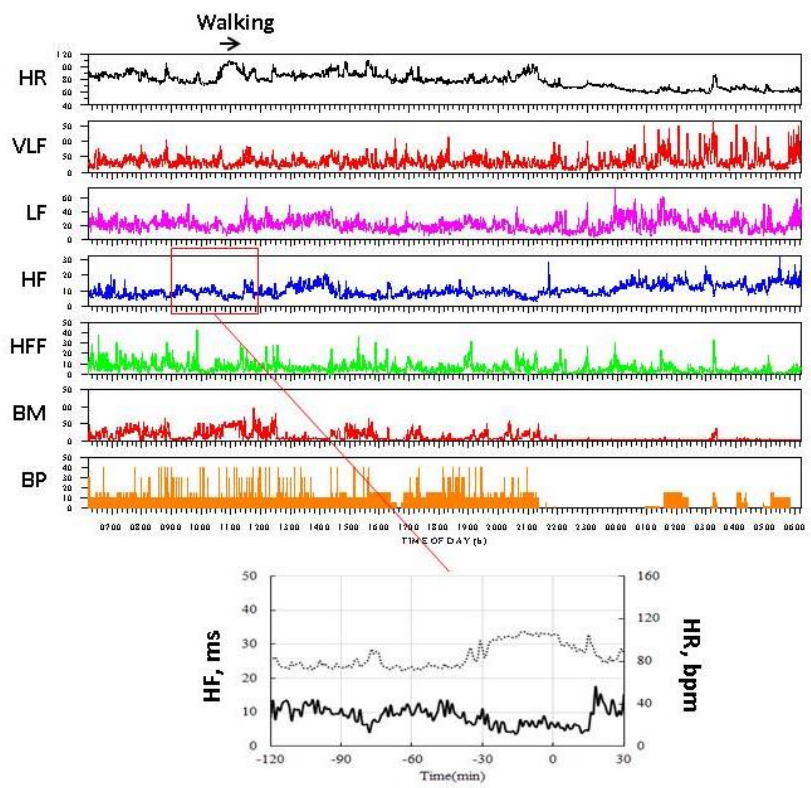

Fig. 4. Heart rate (HR), heart rate variability (HRV), body movement (BM), and body posture (BP) during $24 \mathrm{~h}$ in the same subject as Fig. 2 when she experienced no episode of heatstroke. On this day, she performed treadmill walking between 11:30 and 12:00. HF amplitude shows no spiky HF burst on this day. Abbreviations are explained in legend to Fig. 2.

\section{F. Statistical Analysis}

We used the program package of Statistical Analysis System (SAS, Cary, NC). Effects of age and sex on the frequency of spiky HF burst during $24 \mathrm{~h}$ were evaluated by General Linear model procedure.

\section{RESULTS}

Fig. 2 shows the results of CDM analysis of the $24 \mathrm{~h} \mathrm{HRV}$ and of actigraphic data on the day of golf play with heatstroke. During playing golf normally, the HF amplitude, known as an index of cardiac parasympathetic function, was suppressed $(4.3 \pm 2.2 \mathrm{~ms})$. Whereas, $135 \mathrm{~min}$ before the onset of syncope (14:38), spiky increases in HF amplitude whose SBR <4.0 appeared at an interval of 10-15 $\mathrm{min}$. Then, $51 \mathrm{~min}$ before the onset of syncope, the amplitude of spikes increased to 20-30 ms and spiky HF bursts with SBR > 4.0 appeared at $51 \mathrm{~min}$, $38 \mathrm{~min}, 20 \mathrm{~min}$, and $6 \mathrm{~min}$ before the onset (lower panel of Fig.
2). No such phenomenon was observed during the other part of the day.

Fig. 4 shows the results of CDM analysis in the same subject on the day of walking exercise test (11:30 to 12:00). No sign of heatstroke was observed on this day. Spiky HF burst was observed in HF amplitude neither around this time nor during the other part of the day.

Fig. 5 shows the output from the algorithm developed for detecting spiky HF burst for 24-h data on the days of golf play with heatstroke, walking and running exercise tests, and control. In these graphs, SBR >4.0 was observed repeatedly during the period preceding syncope on the day of golf play with heatstroke, but not at all on the other days without heatstroke.

On the other two days of golf playing without heatstroke in the same subject, spiky HF bursts with SBR >4.0 were observed only once on December 2017 and three times on March 2018 during golf playing, although they were isolated and not repeated during short periods (<15 min).

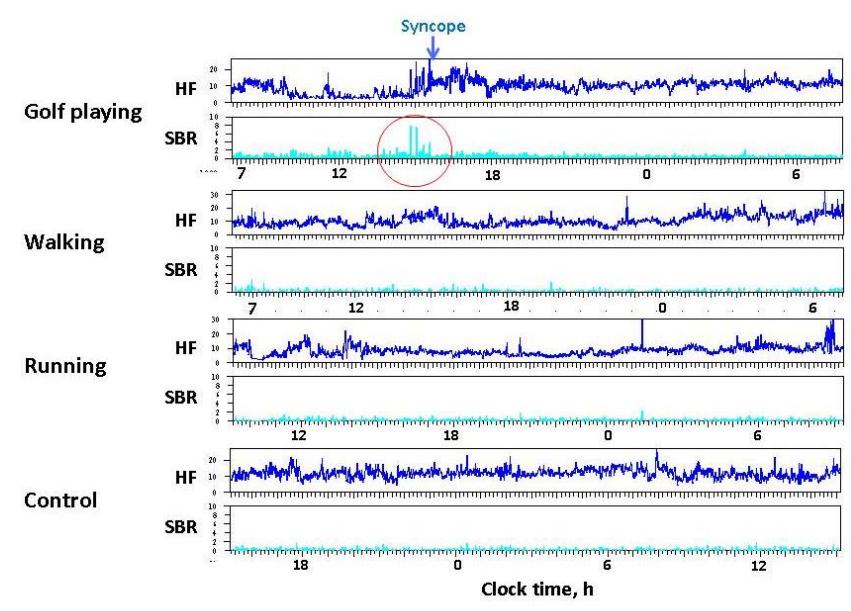

Fig. 5. Spiky HF burst detected by automated algorism in the same subject on four different days of golf play, walking and running exercise, and control (no exercise). Spiky HF bursts was detected preceding NMS episode on the day of golf play but not at all on the other days.

Among 70 subjects in control group, 49 (70\%) subjects showed no spiky HF burst for 24 h, 14 (20\%) showed 1, and 7 (10\%) showed multiple (2-6 times) spiky HF bursts. None of the subjects, however, showed repetitive spiky HF burst within $15 \mathrm{~min}$. There was no significant difference in the frequency of spiky HF burst with age or sex.

\section{DisCUSSIONS}

We analyzed HRV of the 24-h Holter ECG data including an episode of syncope accompanying heatstroke occurred during the summer golf play. We were able to capture a characteristic feature of dynamic changes in the HF component of HRV that preceded the syncopal attack induced by heatstroke. We named the phenomenon as spiky HF burst and defined it as the spiky increase in HF amplitude that has duration about 1 min and amplitude of $4 \mathrm{SD}$ or more than the $\mathrm{HF}$ amplitude of $1 \mathrm{~min}$ before and after the burst. Spiky HF burst thus defined appeared repeatedly at $51 \mathrm{~min}, 38 \mathrm{~min}, 20$ $\mathrm{min}$, and $6 \mathrm{~min}$ before the syncope. Automated software developed to detect spiky HF burst showed that spiky HF 
bursts are not observed for $24 \mathrm{~h}$ on the days of exercise tests or control without heatstroke episodes, whether or not the subject performed exercise. Although isolated spiky HF bursts were observed during golf playing on the other days without heatstroke, they were not repeated during a short period $<15 \mathrm{~min}$. Isolated spiky HF bursts were also observed in $30 \%$ of subjects in healthy control group. None of them, however, showed repetitive spiky HF bursts.

In this study, we used CDM for analyzing the dynamics of HRV components preceding the episode of heatstroke. Because spiky HF burst is a transient dynamic change in HF component within $1 \mathrm{~min}$, it is hard to be detected by power spectral analysis [8]. In a previous study [6], we observed that the time resolution to detect HF amplitude fluctuation by $\mathrm{CDM}$ is $<15 \mathrm{~s}$, which well exceeds the limit achieved by short-segmented spectral analysis.

Our observation is unique from earlier studies that reported characteristics of HRV accompanying NMS [7], [9]. Although it has been known for a long time that the HF amplitude increases as a sign of NMS [7], its practical usefulness as the predictor is quite limited. First, its specificity as the precursor of NMS is low, because HF amplitude increases with deep breathing [10], [11], resting [12], and in any situations where cardiac vagal function increases [8]. Second, it is difficult to set a cutoff value to judgment between normal and abnormal values, because HF amplitude is affected by various physiological and pathological factors, such as age [13], circadian rhythm [14], menstrual cycle [15], and diseases [16], [17]. In contrast, spiky HF burst is defined by the characteristics of the waveform of HF amplitude but not its amplitude, which seem to resolve the weakness of $\mathrm{HF}$ amplitude as the predictor of NMS.

Spiky HF burst is thought to reflect a transient strong increase in cardiac parasympathetic activity. The pathophysiology of syncope accompanying heatstroke is thought to be cardiac vagal activation mediated by the Bezold-Jarisch (BJ) reflex. The BJ reflex originates in cardiac sensory receptors mainly located in the inferoposterior wall of the left cardiac ventricle, which is stimulated by ventricular squeezing caused by ventricular over contraction due to central hypovolemia (reduced venous return) and strong sympathetic stimulation. Spiky HF burst may correspond to incomplete BJ reflex of intensity below the threshold causing syncope.

This is a case-control study with only one case. It is impossible to estimate the extent to which our observation is applicable. Spiky HF burst could be one of prodromal signs of syncope caused by heatstroke, but it should not be thought to be the only sign of it. Our observations in healthy control group indicate, however, that the repetition of spiky HF bursts during short period $<15 \mathrm{~min}$ is specific to the pathologic state that leads syncope due to heatstroke and that it does not appear in normal conditions. These indicate that repetitive spiky HF burst is a specific prodromal sign of syncope accompanying heatstroke but its sensitivity is unknown.

\section{CONCLUSION}

Spiky HF burst is thought to reflect a pathophysiologic state leading syncope accompanying heatstroke. It appears repeatedly only before syncope but not in normal condition. To confirm the usefulness of spiky HF burst as a precursor of heatstroke, verification in the many cases is desirable.

\section{CONFLICT OF INTEREST}

The authors declare no conflict of interest.

\section{AUTHOR CONTRIBUTIONS}

EY conceived the study plan and conduct the study; RI formulated the study concept; YY analyzed the data, and JH developed the software for data analysis and wrote the paper.

\section{REFERENCES}

[1] T. Akihiko, Y. Morioka, and S. K. Behera, "Role of climate variability in the heatstroke death rates of Kanto region in Japan," Sci Rep, vol. 4, p. 5655, Jul 102014.

[2] S. Murakami, N. Miyatake, and N. Sakano, "Changes in air temperature and its relation to ambulance transports due to heat stroke in all 47 prefectures of Japan," J Prev Med Public Health, vol. 45, pp. 309-15, Sep 2012.

[3] M. M. J. Stacey, S. K. Delves, D. R. Woods, S. E. Britland, L. Macconnachie, A. J. Allsopp et al., "Heart rate variability and plasma nephrines in the evaluation of heat acclimatisation status," Eur J Appl Physiol, vol. 118, pp. 165-174, Jan 2018.

[4] L. Rizzo and M. W. Thompson, "Cardiovascular adjustments to heat stress during prolonged exercise," J Sports Med Phys Fitness, vol. 58, pp. 727-743, May 2018.

[5] J. Hayano, J. A. Taylor, S. Mukai, A. Okada, Y. Watanabe, K. Takata et al., "Assessment of frequency shifts in R-R interval variability and respiration with complex demodulation," Journal of Applied Physiology, vol. 77, pp. 2879-2888, 1994.

[6] J. Hayano, J. A. Taylor, A. Yamada, S. Mukai, R. Hori, T. Asakawa et al., "Continuous assessment of hemodynamic control by complex demodulation of cardiovascular variability," American Journal of Physiology, vol. 264, pp. H1229-H1238, 1993.

[7] L. A. Lipsitz, J. Hayano, S. Sakata, A. Okada, and R. J. Morin, "Complex demodulation of cardiorespiratory dynamics preceding vasovagal syncope," Circulation, vol. 98, pp. 977-983, Sep 8, 1998.

[8] J. Hayano, "Introduction to heart rate variability," Clinical assessment of the autonomic nervous system, Japan: Springer, 2016, pp. 109-127.

[9] L. A. Lipsitz, J. Mietus, G. B. Moody, and A. L. Goldberger, "Spectral characteristics of heart rate variability before and during postural tilt: Relations to aging and risk of syncope," Circulation, vol. 81, pp. 1803-1810, 1990.

[10] J. A. Hirsch and B. Bishop, "Respiratory sinus arrhythmia in humans: How breathing pattern modulates heart rate," American Journal of Physiology, vol. 241, pp. H620-H629, 1981.

[11] J. Hayano, S. Mukai, M. Sakakibara, A. Okada, K. Takata, and T. Fujinami, "Effects of respiratory interval on vagal modulation of heart rate," American Journal of Physiology, vol. 267, pp. H33-H40, 1994.

[12] J. Hayano and F. Yasuma, "Hypothesis: respiratory sinus arrhythmia is an intrinsic resting function of cardiopulmonary system," Cardiovascular Research, vol. 58, pp. 1-9, 2003.

[13] D. C. Shannon, D. W. Carley, and H. Benson, "Aging of modulation of heart rate," Am J Physiol, vol. 253, pp. H874-H877, 1987.

[14] J. Hayano, W. Jiang, R. Waugh, C. O'Connor, D. Frid, and J. A. Blumenthal, "Stability over time of circadian rhythm of variability of heart rate in patients with stable coronary artery disease," Am Heart J, vol. 134, pp. 411-8, Sep 1997.

[15] S. Yazar and M. Yazici, "Impact of menstrual cycle on cardiac autonomic function assessed by heart rate variability and heart rate recovery," Med Princ Pract, vol. 25, pp. 374-7, 2016.

[16] J. P. Saul, Y. Arai, R. D. Berger, L. S. Lilly, W. S. Colucci, and R. J. Cohen, "Assessment of autonomic regulation in chronic congestive heart failure by heart rate spectral analysis," American Journal of Cardiology, vol. 61, pp. 1292-1299, 1988.

[17] J. Hayano, Y. Sakakibara, M. Yamada, N. Ohte, T. Fujinami, K Yokoyama et al., "Decreased magnitude of heart rate spectral components in coronary artery disease: Its relation to angiographic severity," Circulation, vol. 81, pp. 1217-1224, 1990. 
Copyright $(\odot 2019$ by the authors. This is an open access article distributed under the Creative Commons Attribution License which permits unrestricted use, distribution, and reproduction in any medium, provided the original work is properly cited (CC BY 4.0).



Emi Yuda was born in Tokyo, Japan in 1980. She studied informatics at Moscow University until 2003 and received her M.S. degree from Tsukuba University, Japan. She will receive the Ph.D. from Nihon University in 2019. From 2013 to 2014 she was a research assistant at the Santa Monica College Computer Science department in California, USA. Since 2015, she has been a project researcher with the Nagoya City University Graduate School of Medical Sciences. Her research is medical informatics and data science. She has many achievements in field of Informatics, computer science and engineering international conference such as IEEE GCCE 2018 (OS-IHP: Informatics of Healthcare \& Health Promotion, Chair), IEEE Life-Tech 2019 (Treasure).

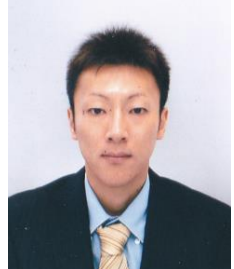

Yutaka Yoshida studied the business administration and computer science at Aichi Institute of Technology and received the Ph.D. degree in 2008. He was a project researcher at Knowledge Hub of Aichi from 2011 to 2015 and was a researcher at Nagoya City University Graduate School of Medical Sciences from 2016 to 2017. Since 2018, he has been a researcher at Nagoya City University Graduate School of Design and Architecture. His specialized field is biological information engineering, signal processing and ergonomics. He received the paper award at the Japan Society of Neurovegetative Research in 2005 and 2007.

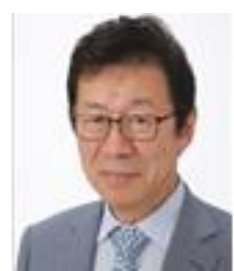

Junichiro Hayano graduated Nagoya City University Medical School, Nagoya, Japan and received M.D. degree in 1980. From 1981 to 1983 , he received residency trainings of psychosomatic medicine in Kyushu University School of Medicine, Fukuoka, Japan. He obtained the Ph.D. degree (Dr. of medical science) in 1988 from Nagoya City University Graduate School of Medical Sciences. From 1990 to 1991, he was working as a visiting associate at the Behavioral Medicine Research Center, Duke University Medical Center, Durham, NC, USA. In 1984, he got a faculty position at Nagoya City University Medical School and has been a Professor of Medicine at Nagoya City University Graduate School of Medical Sciences since 2003. His current interests are applications of dynamic electrocardiography and bio-signal processing to health sciences. 